\title{
LA PRUEBA ILÍCITA. UNA CUESTIÓN DE CONCEPTO
}

\section{Illicit proof. A matter of concept}

Emma Calderón Arias*

Universidad de Girona

Doctoranda en Derecho en la Universidad de Girona, España. Máster en Ciencias Penales y Criminológicas, Licenciada en Derecho por la Universidad de Oriente, Cuba. Docente e investigadora, Facultad de Derecho de la Universitat de Girona, España. Miembro de la Cátedra de Cultura Jurídica. ORCID iD: 0000-0002-9003-5033. Contacto: emma830913@gmail.com 


\title{
Resumen:
}

La prueba ilícita es una institución que se viene tratando desde finales del siglo XIX. La terminología utilizada para su estudio es variada, pero los rasgos de esta institución son comunes a pesar del ordenamiento jurídico en el que se esté aplicando. Por lo general, se define como prueba que resulta de la vulneración de normas jurídicas o, dentro de estas, derechos fundamentales. Sin embargo, en el presente artículo, parto de la posición que entiende la prueba ilícita como resultado de la vulneración de normas del ordenamiento en general. Lo que sucede es que suele abordarse de manera lineal, faltando aun por profundizar en cuestiones específicas de su propio concepto. Ello responde a una especie de inercia y a estudios conjuntos que se muestran en la doctrina cuando se habla sobre la materia. De modo que, se observa la necesidad de realizar una labor de análisis conceptual de la prueba ilícita, incluyendo pautas que desde lo teórico y jurisprudencial que pueden ayudar a enriquecer dichos estudios.

\begin{abstract}
:
Illicit proof is an institution that has been being treated since the late 19th century. The terminology used for its study is varied, but the features of it are common despite the part of the world in which it is developing. It is generally defined as evidence resulting from the infringement of legal rules or, within them, fundamental rights. However, I start from the position that unlawful illicit proof is result of the violation of legal norms in general. What happens is that it usually treated linearly without delving into specific issues of its own concept. This responds to a kind of inertia shown in the doctrine when talking about the concept of it. The need for conceptual clarification of the illicit test noted, including guidelines that from legal theory and jurisprudence can help enrich such studies.
\end{abstract}

Palabras clave:

Prueba - Regla jurídica - Proceso - Ilicitud - Estado de Derecho

\section{Keywords:}

Proof - Rules - Process - Illicit - Rule of law

\section{Sumario:}

1. Ideas iniciales - 2. Apuntes del devenir histórico de la prueba ilícita - 3 . Una aproximación conceptual -4. Los sentidos en que se define la prueba ilícita - 5. La prueba ilícita como producto de la violación de derechos 
fundamentales - 6. La prueba ilícita como producto de la violación de normas jurídicas -7 . Conclusiones -8 . Lista de referencias

\section{IDEAS INICIALES}

La prueba ilícita es tratada - usualmente - por la doctrina y la jurisprudencia desde un enfoque relativo a la exclusión como una de sus principales consecuencias. Al abordarse desde este punto de vista, se está dejando de lado una parte que también puede ser interesante. Con ello, se hace referencia al estudio relativo al concepto de esta institución de conjunto con los problemas que devienen de este.

Pues bien, un trabajo que logre separar el concepto sin ser construido este necesariamente desde las consecuencias puede ayudar a esclarecer muchas de las confusiones que se presentan al debatir sobre prueba ilícita. Con este artículo, justamente, se pretende seguir la mencionada línea para llegar a explicar mejor los casos de prueba ilícita.

Uno de los problemas fundamentales que se puede observar es que se ha extendido a gran parte del mundo lo que se conoce como prueba ilícita desde una óptica propia de los autores estadounidenses. Este enfoque, que nace y se desarrolla precisamente a partir de la exclusión de prueba que ha sido obtenida en contra de los derechos fundamentales, tiene otras aristas que se han venido estudiando a lo largo de los años y que explican otros tipos de casos no relativos necesariamente a la violación de dichos derechos.

En ese sentido, se detraen pruebas que, aunque han sido afectadas por ilicitud en su obtención o práctica, pero ya no solo mediante violación de derechos fundamentales, sino, también, a las que resulten de infracción de otras normas del ordenamiento jurídico. Este punto, quizás, sea uno de los que corresponda aclarar en el análisis conceptual, junto a otros debates que siguen estando presentes y en los que vale la pena detenerse.

Otro problema que se presenta es la terminología que se utiliza cuando de prueba ilícita se trata. Los usos de esta expresión o término son diversos y tienden a confundir. En ocasiones, no se identifica exactamente de qué se está hablando. Al mismo tiempo, existen otras cuestiones de importancia a debatir referidas a los sentidos en que es definida, como genuino problema conceptual, punto esencial de este trabajo en cuestión.

Las normativas de muchos de los países de Iberoamérica reflejan el enfoque desde el cual se define la prueba ilícita, pero al mismo tiempo se denota 
un proceso de positivación de dicha institución, que, si nos fijamos, no estaba presente antes del siglo XXI. Todo ello nos da pie para ahondar en el estudio que se pretende.

A continuación, nos detendremos en conocer de dónde vienen los orígenes de la prueba ilícita, para luego enfocarnos en el análisis del concepto como tal como principal aporte del presente trabajo en cuestión.

\section{APUNTES DEL DEVENIR HISTÓRICO DE LA PRUEBA ILÍCITA}

¿Cuándo nace la prueba ilícita? Pues bien, el origen de esta se puede encontrar en Estados Unidos de América con el análisis de algunos casos en el orden jurisprudencial. Dichos casos trataban sobre acciones relativas a incautaciones arbitrarias por parte de la policía en asuntos de índole civil y penal ${ }^{1}$.

Esta doctrina adquirió auge en la primera mitad del siglo XX². Precisamente, cuando la Corte Suprema Federal estadounidense declaraba la nulidad de los juicios en procesos penales donde se habían ejecutado acciones de registros, ocupación de documentos o detenciones sin autorización judicial contrarias a los derechos de los ciudadanos ${ }^{3}$.

Por su parte, en Inglaterra surgió la disciplina de la prueba ilícita en el análisis de casos donde se valoró que la confesión arrancada bajo presión no tenía validez alguna para el proceso. La idea que se presentaba era que no podía tener fuerza probatoria alguna una declaración tomada bajo presión o coacción, pues esto anulaba en cierta medida la credibilidad de lo que se podía declarar por parte del acusado, por ejemplo; además, de ir en contra de los derechos de este 4 .

De otro lado, en la doctrina alemana, fue Beling ${ }^{5}$ quien trató las prohibiciones probatorias como límites a la averiguación de la verdad en el proceso penal,

1 Casos: Boyd v. United States, 116 U.S. 616 (1886) y Bram v. United States, 168 U.S. 532 (1897).

2 Derivación de la jurisprudencia citada en la nota anterior, fue la doctrina de los frutos del árbol envenenado, que surge en 1920 con el caso Silverthorne Lumber Co. v. U.S.; confirmándose dicha denominación en el caso Nardone v. U.S. de 1939.

3 En las decisiones de casos como: Weeks v. United States, 232 U.S. 383 (1914); Gouled v. United States, 255 U.S. 298 (1921); Agnello v. United States, 269 U.S. (1925).

4 Roe, Haldane, Urry v. Havey, 1769. Burrow, 1790: 2484-2486: "in a criminal or penal cause, the defendant is never forced to produce any evidence; though he should hold it in his hands, in Court" (2484-2486). Además, se puede encontrar de manera más clara en el caso: The King v. Rudd, 1775: "If any evidence or confession has been extorted from her, it will be of no prejudice to her on the trial". Leach, 1815, p. 123. Véase Nieva (2017, p. 3).

5 Beling distinguía entre: prohibiciones de producción probatoria y prohibiciones de utilización de pruebas. 
categorías por cierto muy utilizadas en la doctrina actual $\left(1903\right.$, p. 26) ${ }^{6}$. En ese entonces en Inglaterra y Alemania, se planteó este tema bajo el fundamento de la determinación de excepciones a las facultades de investigación del Estado, positivándose así las prohibiciones probatorias (Guariglia, 1996, pp. 76-77).

Las diferentes formas de plantear el tema de la prueba ilícita se evidencian desde la alusión que la doctrina hace sobre los referentes históricos; por un lado, se trata la exclusión de esta bajo el fundamento de la disuasión; y, por otro lado, se enfoca el problema a partir de las prohibiciones probatorias como límites a la averiguación de la verdad. Las polémicas que se han ido desarrollando sobre el tema dependen de la perspectiva que se asuma (exclusión de la prueba ilícita o prohibiciones probatorias).

\section{UNA APROXIMACIÓN CONCEPTUAL}

El análisis de aclaración conceptual acerca de la prueba ilícita resulta necesario, puesto que, aunque se han efectuado muchas investigaciones sobre el tema (desde la dogmática civil ${ }^{7}$, penal ${ }^{8}$, etc., y también desde una vasta jurisprudencia al respecto ${ }^{9}$ ), sigue siendo confusa la forma de concebirla y muchas veces apresurada la conexión entre ilicitud probatoria y el efecto de la exclusión. Un estudio de este tipo ayudará a una mejor presentación del tema, clarificando varios de sus elementos referidos a los sentidos que se le vinculan a esta.

Las principales dificultades relativas al concepto de prueba ilícita están dadas por la vaguedad de este visto desde los sentidos que en la doctrina y en

6 Comparto el criterio de Gómez Colomer (1985, p. 133), específicamente en la nota número 26, para quien la expresión "prueba prohibida" es una traducción incorrecta del alemán (que han realizado muchos), pues la doctrina de Beling, E., en Die beweisverbote als grenzen der Wahrheitserforschung im Strafprozess, fue emplear dichos términos siempre en plural, refiriéndose a prohibiciones de prueba, dado que existen varios supuestos. De todas formas y, como bien apunta dicho autor, es una denominación cómoda y gráfica para tratar aquellos supuestos en los que la prueba es inadmisible.

7 Picó i Junoy (1996, p. 290); Passanante (2017, p. 63); Peláez Hernández (2017, p. 379).

8 Guariglia (2005, p. 76); Nieva Fenoll (2017, p. 5); Miranda (2018, p. 18); Armenta Deu (2007, p. 2).

9 Algunos ejemplos de jurisprudencia se pueden ubicar en Estados Unidos: Weeks v. United States, 232 U.S. 383 (1914) y Mapp v. Ohio, 367 U.S. 643 (1961). En España, las que se referencian a continuación: Tribunal Constitucional, Sentencia N 114/1984, Sentencia N²22/2012 de 27 de noviembre, Sentencia $N^{\circ}$ 54/2015 de 16 de marzo, Sentencia $N^{\circ}$ 61/2021 de 15 de marzo; del Tribunal Supremo, Sentencia N 9350/1991 de 16 de diciembre, Sentencia № 817/2012 de 23 de octubre, Sentencia $N^{\circ} 3170 / 2016$ de 5 de julio, Sentencia $N^{\circ}$ 2286/2018 de 14 de junio, Sentencia $N^{\circ} 3191 / 2020$ de 14 de octubre, Sentencia $N^{\circ}$ 4446/2020 de 3 de diciembre y Sentencia $N^{\circ} 1157 / 2021$ de 24 de marzo. 
la dogmática se le adscriben. Como ya se planteaba, centraremos la atención en el problema de los sentidos en que se define prueba ilícita, como genuino problema conceptual; pues, al final, la terminología a utilizar formará parte de la definición que se asuma.

\section{LOS SENTIDOS EN QUE SE DEFINE LA PRUEBA ILÍCITA}

Lo que se entienda en general por prueba ilícita es el punto esencial en el análisis conceptual que resulta necesario desarrollar. El mejor modo de responder este interrogante es distinguiendo enfoques que plantean argumentos distintos. La presentación de los enfoques tiene como fin examinar la cuestión conceptual sobre qué se concibe como prueba ilícita a partir de un estudio de la doctrina, la dogmática y la jurisprudencia ${ }^{10}$.

El primero de los enfoques concibe a la prueba ilícita considerando que es la prueba obtenida mediante violación de derechos fundamentales; y el segundo, plantea que es aquella que resulta de la vulneración de normas del ordenamiento jurídico y su impacto en el proceso. En el siguiente espacio me referiré a la primera posición, específicamente, la que define prueba ilícita como producto de la violación de derechos fundamentales. Entre los debates esenciales se abordarán puntos tales como: i) la concepción general de derechos fundamentales, o ii) el problema de identificación de los derechos fundamentales en su aplicación a casos concretos de prueba ilícita durante la actividad probatoria.

\section{LA PRUEBA ILÍCITA COMO PRODUCTO DE LA VIOLACIÓN DE DERECHOS FUNDAMENTALES}

Para un sector de la doctrina, la prueba ilícita se entiende como aquella prueba que se obtiene mediante violación de derechos fundamentales ${ }^{11}$. Uno de los principales exponentes de esta postura es Miranda Estrampes (2010),

10 Algunos ejemplos de la jurisprudencia estudiada: Boyd v. United States 116 U.S. 616 (1886); Weeks v. United States, 232 U.S. 383 (1914) y Mapp v. Ohio, 367 U.S. 643 (1961). De España, las siguientes: del Tribunal Constitucional, Sentencia N 114/1984 de 29 de noviembre; del Tribunal Supremo, Sentencia N²647/1990 y N 9350/1991. Así como, las sentencias de los casos Al'Khawaja and Tahery v. UK (2009), y Seton v. UK (2016) del Tribunal Europeo de Derechos Humanos (en lo adelante, TEDH).

11 Posición seguida, entre otros, por: Gascón Abellán (2006, pp. 56-58); Picó i Junoy (1996, p. 286); González (1990, p. 31); Asencio (1986, pp. 37-38); Tomé (1993, p. 468); Martínez (2003, p. 38); Arozamena (1999, p. 83); De Diego Diez (2001, p. 8); Ramos (2000, p. 20); Parra (2002, p. 39). 
quien define la prueba ilícita de la siguiente manera: "por prueba ilícita debe entenderse aquella prueba obtenida y/o practicada con vulneración de derechos fundamentales" (p. 133).

Este punto de vista se sustenta en dos argumentos, por un lado, el que se refiere a la defensa del ideal del Estado de Derecho; y, por otro lado, el que plantea el derecho a utilizar medios de prueba pertinentes para la defensa. El primero de estos argumentos se basa en el ideal del Estado de Derecho, perspectiva que arguye que existen razones para declarar una prueba como ilícita en tanto:

Desde planteamientos democráticos es inadmisible o muy difícil asumir como válidos los resultados derivados de actos contrarios a los derechos fundamentales. No hacerlo así, y considerarlos lícitos, sería tanto como reducir a mero formalismo su eficacia, puesto que la respuesta penal, civil o disciplinaria frente a los autores de actos contrarios a derechos fundamentales no es totalmente satisfactoria y, por supuesto, no garantiza la idoneidad de una investigación (Asencio, 1990, p. 246).

Este primer argumento se sostiene en la denominada concepción democrática de los derechos fundamentales ${ }^{12}$, para la cual esos derechos tienen también un aspecto democrático. Así lo afirma Bustamante Alarcón cuando dice lo siguiente:

Los derechos fundamentales además de tener un aspecto personal y privado, tienen un aspecto democrático y público que los convierte en condición misma de la democracia, pues sin su reconocimiento y ejercicio la democracia no puede existir como sistema político (2018, p. 62).

Esta posición defiende una determinada noción del Estado democrático de Derecho en la que los derechos fundamentales constituyen un elemento esencial. La presencia de derechos fundamentales caracteriza el funcionamiento de un específico modo de articulación y ejercicio del poder político por parte del Estado democrático de Derecho. Asencio se refiere a la "validez" como uno de los aspectos de especial importancia a tener en cuenta en relación con los actos que resultan de la violación de derechos fundamentales. La validez va de la mano con la legitimidad en este sentido, y ello se debe, en parte, a la propia prohibición que tiene el Estado de violar esos derechos fundamentales. Un sistema democrático no debe permitir actos contrarios a los derechos

12 Véase Häberle (1997, p. 71). 
fundamentales, ya que esos derechos son uno de los componentes esenciales del mismo ${ }^{13}$.

Ahora bien, traer este tema específico de la defensa del Estado democrático de Derecho a los debates sobre prueba ilícita, supone que es la vulneración de alguno de esos derechos lo que resulta en una prueba ilícita. El punto en discusión acá es si se deberían o no calificar como válidas las pruebas que resulten de actos contrarios a esos derechos fundamentales, pues entenderlas como tal, afectaría ese ejercicio de la democracia, y con ello se afectarían también las bases del Estado democrático de Derecho. Miranda Estrampes resume estas ideas afirmando que el Estado democrático de Derecho no puede permitirse el hecho de admitir y valorar pruebas obtenidas con vulneración de derechos fundamentales, pues eso sería ir en contra de las propias garantías constitucionales que constituyen los pilares básicos sobre los que se asienta $(2018$, p. 36).

La prueba ilícita entendida desde esa perspectiva se basa en argumentos que, como se puede notar, son de índole jurídico. Dichos argumentos tienen en cuenta la importancia y el papel esencial que tienen las normas jurídicas en la definición de un Estado democrático de Derecho. En resumen, lo que se argumenta es que la transgresión de una norma constitucional que expresa un derecho fundamental supone la invalidez jurídica del acto en virtud de la jerarquía normativa de la Constitución.

Pues bien, estas ideas relativas al Estado democrático de Derecho y que apoyan el primero de los argumentos, tienen como trasfondo el razonamiento en defensa de la exclusión de la prueba ilícita. De modo que, toda prueba que resulte de la violación de los derechos fundamentales afectaría el ejercicio de la democracia, pudiéndose justificar de esa manera su exclusión.

El segundo argumento (a favor de la posición que entiende la prueba ilícita como resultado de la violación de derechos fundamentales) tiene como fundamento el derecho a utilizar los medios probatorios pertinentes para la defensa. Es decir, no son pertinentes aquellas pruebas que hayan sido obtenidas mediante actos que violen derechos fundamentales, porque las pruebas que resulten de esos actos violatorios serán declaradas como ilícitas (ilícitas en el sentido de que ignoran las garantías procesales). Picó i Junoy (1997) es uno de los principales exponentes de este punto de vista, y así lo expresa en las siguientes afirmaciones:

13 Compárese con Pérez Luño (2017, p. 669); Martínez-Sicluna (1991, p. 49); Monereo (2006, p. 101). 
La prueba ilícita es aquella obtenida o practicada con infracción de derechos fundamentales, restricción cuyo fundamento se encuentra en el derecho a utilizar los medios probatorios pertinentes para la defensa. En los supuestos de prueba ilícita se ignoran las garantías procesales, y se introduce desigualdad entre las partes, lo cual impide considerar pertinente un elemento de prueba así obtenido (p.147).

Este autor opta por una concepción que sólo considera ilícita la prueba obtenida en violación de derechos fundamentales (y no una concepción más amplia que considere ilícita toda prueba obtenida en violación de normas), puesto que lo que pretende es encontrar un equilibrio con el derecho a la prueba. Es decir, como desde su punto de vista la ilicitud conlleva la exclusión, le parece que no se puede excluir toda prueba obtenida en violación de normas (porque afecta al derecho a la prueba). Esto, en realidad, es una buena muestra de los problemas de tratar conjuntamente el problema conceptual y el de las consecuencias.

De este segundo argumento expuesto por Picó i Junoy quisiera destacar lo siguiente: en primer lugar, es un razonamiento más específico que el que se planteó con anterioridad (el que defiende el Estado democrático de Derecho con base en planteamientos políticos). La especificidad está dada en que se sostiene con base en algunos de los derechos y/o garantías (concretamente, el debido proceso como garantía procesal que se vincula a la prueba ilícita,) como parte de la idea general del Estado democrático de Derecho. El debido proceso, en conjunto con el principio de igualdad de las partes y el derecho a la defensa, son algunos de los elementos involucrados en la "faceta democrática" a la que se aludió. Por tanto, se podría ver también atacado ese ejercicio de la democracia una vez que se violen estos.

En segundo lugar, este argumento se puede considerar como un entimema, ya que le está faltando la mención al debido proceso como una de las premisas fundamentales de las que parte. En este sentido, se da por sobreentendido "el debido proceso como garantía procesal", y en caso de ser ignorada dicha garantía, se puede producir una afectación al principio de igualdad de las partes y, con ello, el derecho a la defensa. Dicha afectación a la igualdad y al derecho a la defensa (teniendo en cuenta que, para una parte de la doctrina, estos forman parte del debido proceso) traería consigo que una prueba obtenida de ese modo no sea considerada como pertinente. La pertinencia entendida como criterio que delimita el derecho de las partes a aportar pruebas relacionadas con los hechos en el proceso, y a su vez, debe concebirse como uno de los elementos del contenido del derecho a la prueba. 
Ahora bien, ¿̇uál ha sido el reflejo de este enfoque en la jurisprudencia? El análisis jurisprudencial aporta información relevante de cómo han decidido los órganos jurisdiccionales al respecto y dice, además, cuáles son los principales aspectos que se debaten en ese orden.

La sentencia $\mathrm{N}^{\circ} 114 / 1984^{14}$ es un ejemplo de cómo se refleja en la jurisprudencia esta posición referida a la prueba ilícita como resultado de la violación de derechos fundamentales, y cito:

(...) Hay que reconocer la posición preferente de los derechos fundamentales en el ordenamiento y de su afirmada condición de «inviolables», con la imposibilidad de admitir en el proceso una prueba obtenida violentando un derecho o una libertad fundamental. Para nosotros, en este caso, no se trata de decidir en general la problemática procesal de la prueba con causa ilícita, sino, más limitadamente, de constatar la «resistencia» frente a la misma de los derechos fundamentales (...).

Esta afirmación en relación con la posición preferente de los derechos fundamentales y su condición de inviolables es una de las razones por las cuales se asevera que no es posible admitir una prueba obtenida como resultado de la violación de dichos derechos. Ello nos obliga a volver al punto donde se explicó que el argumento detrás de este tipo de posiciones es el que se refiere a la exclusión de la prueba ilícita (en otras palabras, los posibles efectos de la prueba ilícita) cuando esta resulta de la violación de derechos fundamentales. En cambio, parece oportuno distinguir el análisis conceptual de la prueba ilícita y de sus fundamentos, para luego centrar la atención en los efectos que produce la misma.

La sentencia citada es considerada como una de las sentencias más significativas en España, pues marcó un antes y un después con relación al debate sobre el tratamiento de la prueba ilícita ${ }^{15}$. A partir de este pronunciamiento, se ha desarrollado una extensa polémica que versa sobre la tensión existente entre los derechos fundamentales y la prueba ilícita, así como el punto relativo a cuáles de estos derechos han de vulnerarse para que una prueba sea considerada como tal. Dicha polémica ha dado lugar a que esta sentencia se encuentre

14 Sentencia del Tribunal Constitucional de España del 29 de noviembre de 1984.

15 Marca un antes y un después porque, previo a que saliera a la luz esta sentencia, la legislación española no tenía establecida la prueba ilícita y los efectos de esta. Después de dicha sentencia, se aprobó el artículo 11.1 de la LOPJ de España, el cual establece la regla de exclusión de las pruebas ilícitas. Véase Martínez (2003, pp. 21-22); Fidalgo (2003, pp. 17-20); Alday (2020, pp. 54-59). 
citada por muy diversos autores ${ }^{16}, y$, además, a que haya sido seguida en otros tantos pronunciamientos jurisprudenciales ${ }^{17}$.

A pesar de lo expuesto hasta aquí sobre la prueba ilícita como producto de la violación de derechos fundamentales, no es claro si la prueba sería ilícita como resultado de la violación de cualquier derecho fundamental o sólo de algunos de ellos. Sobre este particular, no basta con decir que "es prueba ilícita la que resulta de la violación de derechos fundamentales" y abordar sutilmente algunos puntos discutibles de esta posición. El aspecto en el que se puede ganar en precisión es la determinación de cuáles son los derechos fundamentales cuya vulneración hace que una prueba sea ilícita.

Para responder este interrogante habría que, como un primer punto de discusión, analizar la concepción general de los derechos fundamentales, aún sin desarrollar una teoría robusta y sofisticada sobre estos; pero sí resulta importante saber cuáles son los criterios de identificación de esos derechos para que resulte una prueba como ilícita. Este punto de discusión ayudará al esclarecimiento de varias de las ideas que se debaten sobre el tema de los derechos fundamentales y su conexión con la prueba ilícita.

Una parte de la doctrina sostiene que los derechos fundamentales son todos aquellos derechos reconocidos en el texto constitucional ${ }^{18}$. Así lo afirma, por ejemplo, Gálvez Muñoz cuando plantea que "los derechos fundamentales son todos los contemplados en la Constitución"19. De seguirse esta posición, una prueba se podría declarar como ilícita cuando en su obtención se viole cualquiera de los derechos reconocidos constitucionalmente ${ }^{20}$.

16 Asencio (1985, p. 289); Pomarón (1989, pp. 1 y ss.); Bandrés Sánchez-Cruzat (1992: 395); Miranda (2013, p. 27); Gálvez (2003, p. 74).

17 Entre esos otros pronunciamientos están: del Tribunal Constitucional, Sentencia N 107/1985 de 7 de octubre, Auto de la Sala Segunda del Tribunal Supremo de 18 de junio de 1992 ("Caso Naseiro"), Sentencia N 111/2011 de 4 de julio, Sentencia Nº 54/2015 de 16 de marzo, Sentencia $N^{\circ} 116 / 2017$ de 23 de febrero (Caso "Lista Falciani"), Sentencia N 97/2019 de 16 de julio y Sentencia N61/2021 de 15 de marzo.

18 Gálvez (2003, pp. 81-82); Cruz (1989, p. 35).

19 Además, pone como ejemplo a los derechos subjetivos reconocidos en el Título I de la Constitución española. Para este punto de vista quedan fuera los principios rectores de la política social y económica previstos en el Capítulo III de la propia Constitución (Gálvez, 2003, pp. 81-82).

20 Sobre el tema de los derechos fundamentales, existen autores que conciben una lista más o menos amplia de estos derechos, por ejemplo: Gálvez Muñoz señala que "deben incluirse todos los establecidos en el capítulo segundo del texto constitucional español, de artículo 14 al 38" (2003, pp. 81-82). Mientras que, para otros, son derechos fundamentales los que se encuentran recogidos en la sección primera del capítulo segundo del Título I de la propia Constitución de 
Frente a esta postura, Peces-Barba $(1995$, p. 469) y otros autores sostienen que no todo derecho reconocido en la Constitución es un derecho fundamental $^{21}$. Desde esta perspectiva, y siguiendo una postura hohfeldiana, los derechos fundamentales se definen como derechos subjetivos, libertades, potestades o inmunidades ${ }^{22}$. Esta postura restringe el ámbito de aplicación de la prueba ilícita si es que la misma resultaría solamente con la vulneración de derechos fundamentales. La prueba ilícita entendida de esta forma implicaría que esta puede resultar solamente de la violación de esos derechos fundamentales y no de otros derechos $u$ otras normas, pudiendo quedar fuera posibles casos.

Dentro de todo este debate relativo a la tensión existente entre prueba ilícita y derechos fundamentales, otro de los puntos que se discute en la doctrina es en lo relativo a qué entender por violación de derechos fundamentales. La discusión aquí se refiere a cómo se puede considerar violado un derecho fundamental. Las respuestas se plantean en dos sentidos, por un lado, los que entienden que se viola un derecho fundamental únicamente en caso de vulnerarse su contenido constitucional (González-Cuellar, 1990, pp. 337-339), y, por otro lado, quienes defienden la idea de que debe incluirse también la infracción de sus normas de desarrollo (Díaz Cabiale y Morales, 2001, p. 28).

Este debate sobre cómo se puede considerar violado un derecho fundamental encuentra respuestas también en la jurisprudencia. El Tribunal Constitucional español se ha pronunciado al respecto y se decanta por la solución que se limita a entender la violación de derechos fundamentales como la violación de su regulación constitucional y no de sus normas de desarrollo (Gálvez, 2003, p. 83).

Otro de los puntos problemáticos que interesa tratar es la cuestión relativa a si la prueba ilícita puede resultar de la violación de cualquier derecho fun-

España (entre los artículos 15 al 29), así como el principio de igualdad reconocido en el artículo 14 del propio texto constitucional.

21 Cuestión diferente es la polémica sobre si es posible jerarquizar o no los derechos fundamentales. Algunos autores entienden que sí hay derechos superiores a otros. Y lo plantean de esa manera con el argumento de que, estos derechos son superiores porque funcionan como presupuesto básico para el mantenimiento de la democracia (Currie, 1987, p. 43; Stonecipher, 1990, p. 17; y González, 1990, p. 1). Sin embargo, otra parte de la doctrina opina que no es posible jerarquizarlos, en tanto son inherentes al ser humano, y no tendría sentido poner unos por encima de otros (Gálvez, 2003, p. 83).

22 (Peces-Barba, 1995, p. 462). A la par que se valora como rasgos esenciales de los derechos fundamentales la tutela judicial y el respeto de su contenido esencial por el legislador, por tanto, son derechos fundamentales sólo los derechos que tengan esas características y estén reconocidos en el texto constitucional. 
damental. Pues bien, para una parte de la doctrina, la prueba ilícita es la que resulta de la violación de algunos derechos fundamentales, específicamente los que se denominan como "derechos procesales" ${ }^{23}$. Los seguidores de esta posición expresan que algunos derechos fundamentales tienen incidencia en el proceso y son sólo estos los que se conectan con la prueba ilícita (y ello en consonancia con que se entienda que la prueba ilícita sólo tiene lugar en el contexto procesal, de todos modos, este particular se tratará en un epígrafe más adelante). Desde mi punto de vista, esta postura tiene un inconveniente de peso y es que el derecho a la intimidad o al secreto de las comunicaciones, por ejemplo, son derechos que pueden verse violados en casos típicos de pruebas ilícitas y, en cambio, no son derechos procesales.

La contraparte de esta posición entiende que la prueba ilícita sí puede resultar de la violación de cualquier derecho fundamental, sin hacer distinciones entre los derechos procesales o sustantivos, puesto que, desde un punto de vista los derechos fundamentales están presentes tanto fuera como dentro del proceso. Se podría decir entonces que, si la prueba ilícita se puede ubicar tanto en el contexto procesal como en el extraprocesal ${ }^{24}$, ello indica en parte, que la misma puede resultar de la violación de derechos fundamentales (entendiendo estos en el sentido que lo plantea la postura hohfeldiana), no solamente de los de índole procesal. Esta es la posición que comparto, pues no creo sea adecuado, en este contexto, clasificar los derechos fundamentales por categorías sino, que se deben tratar todos por igual. Siendo así, se pueden analizar casos donde se violan derechos fundamentales de los que llaman sustantivos y de esa violación resultar una prueba ilícita (violación del derecho al secreto de las comunicaciones, por ejemplo), así como se pueden vulnerar derechos fundamentales de los que denominan como procesales y también resultar una prueba ilícita.

Ahora bien, sigue siendo necesaria la precisión relativa a: ¿es prueba ilícita solamente la que resulta de la violación de derechos fundamentales? En la doctrina existen posiciones encontradas al respecto, por un lado, hay autores que afirman que $s^{25}$, que la prueba ilícita es solamente la que resulta de la violación de derechos fundamentales. En correspondencia con ello, se encuentra la distinción que realiza Miranda Estrampes (anteriormente aludida), para

\footnotetext{
23 Para profundizar en el tema de los derechos procesales: Jaén Vallejo (2007, p. 6); Armenta Deu (2009, p. 37); Díez-Picazo (1996, p.122); Oliva Santos (1980, p. 132).

24 Miranda Estrampes (2013, p. 74; 2018, p. 53); Martínez (2003, p. 18).

25 Miranda Estrampes (2013, p. 61); Gascón Abellán (2006, p. 47); Picó i Junoy (1996, p. 290).
} 
quien prueba ilícita y prueba irregular son conceptos diferentes, concibiendo a la primera como aquella prueba que resulta de la violación de derechos fundamentales, y la prueba irregular es la que resulta de la vulneración de normas de carácter ordinario.

Esta es una distinción que interesa a los fines de este trabajo puesto que lo que para este autor es una categoría de prueba diferente de la prueba ilícita (denominada como "prueba irregular"), para otros autores sigue siendo prueba ilícita. La contraparte de la posición anterior es esta otra forma de entender prueba ilícita, como aquella que resulta de la violación de cualquier regla del ordenamiento jurídico, sean estas reguladoras de derechos fundamentales u otras reglas ${ }^{26}$.

Los ejemplos ayudarán a explicar mejor el punto, pues creo que ello resulta necesario para ganar claridad sobre este punto relativo a si la prueba ilícita puede resultar solamente de la violación de derechos fundamentales. Veamos un primer ejemplo: " $X$ " graba las conversaciones telefónicas de " $Y$ " sin autorización judicial, y la prueba obtenida (la grabación de esa conversación telefónica) es declarada como ilícita. Un segundo ejemplo: el agente de la policía "Z" realiza un registro al domicilio de "W" sin autorización judicial y sin la presencia de dos testigos (este requisito de la presencia de dos testigos está dispuesto en la ley). En dicho registro se ocupan drogas y armas de fuego.

Ahora bien, en ambos casos hay prueba ilícita como resultado de la violación de un derecho fundamental (el secreto de las comunicaciones en el primer ejemplo, y el derecho a la inviolabilidad del domicilio en el segundo). Sin embargo, en el segundo ejemplo, la diferencia está en que, además de la violación del derecho fundamental, está la infracción de uno de los requisitos legales establecidos para el registro. La infracción de dicho requisito influye directamente en el hecho de que podamos fiarnos o no de esas pruebas. Más allá de la ilicitud, existe una afectación en la fiabilidad.

El punto es si en el caso que resulte una prueba de la violación de derechos fundamentales puede suceder que ésta no sea ilícita. Esto depende del concepto de prueba ilícita. Claro, porque si entendemos prueba ilícita como la obtenida o practicada en violación de derechos fundamentales, entonces por definición una prueba obtenida así siempre será ilícita.

Se sigue de este análisis lo planteado por Díaz Cabiale y Martín Morales (2001) cuando afirman lo siguiente:

26 Véase Martínez García (2003, p. 81); Sainz y Albacar (1983, p. 600); Pastor Borgoñón (1998, p. 348). 
Hay prueba ilícita cuando existe lesión de un derecho fundamental, pero no siempre que un derecho fundamental resulta menoscabado en una actuación probatoria cabe hablar de prueba ilícita. Por ejemplo, si se da lectura en el juicio oral a un informe pericial, llevado a cabo en el procedimiento preliminar sin que la defensa pudiera intervenir, y sin que ahora el experto esté presente en la vista, es evidente que no se permite la contradicción y se lesiona el derecho a la defensa. Hay lesión de un derecho fundamental y se trata de actividad probatoria, pero no hay prueba ilícita (p. 22).

Dichos autores plantean la idea de que no toda violación de derecho fundamental trae consigo una prueba ilícita. Sin embargo, considero que el caso que utilizan como ejemplo no es el más adecuado para explicar el problema, ya que estaría faltando la precondición de la irregularidad de la prueba. Si hay irregularidad y violación de derechos fundamentales hay prueba ilícita y no es infraincluyente.

Además, creo que esto depende de si se restringe la prueba ilícita a los supuestos de violación de derechos fundamentales en su obtención o, también, en su práctica. Con la idea de Miranda Estrampes ${ }^{27}$ de que es prueba ilícita también aquella en la que se han violado derechos fundamentales en la práctica de la prueba, el ejemplo de Díaz Cabiale y Martín Morales sería una prueba ilícita.

Todos los debates tratados hasta el momento sobre prueba ilícita y los derechos fundamentales apuntan a que el problema no es el fundamento en sí de los derechos fundamentales, sino que, como explicación de todo el fenómeno de la prueba ilícita, esta es una posición que no llega a explicar todos los tipos de casos. Ello indica que este criterio relativo a la prueba ilícita como producto de la violación de derechos fundamentales es un criterio infrainclusivo. Tal como plantean algunos autores, hay casos de prueba ilícita que no quedan incluidos aquí, pero esto depende de la definición.

La posición que concibe a la prueba ilícita como resultado de la violación de derechos fundamentales presenta el problema de que no explica todos los casos de prueba ilícita porque hay casos en donde no hay violación de derechos fundamentales, y puede valorarse una prueba ilícita. Es decir, se quedan casos sin fundamentar con ese criterio, por tanto, es un criterio infra-

27 Dicho autor afirma que la vulneración de derechos fundamentales puede tener lugar no solo en el momento de la obtención de la prueba, sino también en el momento de su incorporación y producción en el proceso. Dentro de estas últimas se encuentran aquellas pruebas en cuya práctica no se han respetado las garantías constitucionales de contradicción, oralidad, publicidad e inmediación impuestas por ley y conectadas con el derecho fundamental a la presunción de inocencia $(2018$, p. 81). 
inclusivo. Sin perjuicio de ello, también se podría decir que es una cuestión de definición.

Otro aspecto que resulta importante tratar es que, si nos referimos a la definición de prueba ilícita, hay una de las que se ofrece que está basada en la violación de derechos fundamentales. No obstante, además, hay otras definiciones de prueba ilícita con base en la violación de normas jurídicas, e incluso de tipos específicos de éstas. La posición que define la prueba ilícita como resultado de la violación de derechos fundamentales no abarca todos los casos posibles, pero como criterio sí es informativo para explicar ciertas reglas específicas, como son los supuestos de exclusión de la prueba ilícita, pues es fundamento de la regla de exclusión, por ejemplo.

En resumen, defiendo una posición según la cual:

i. Los derechos fundamentales son solo algunos de los que aparecen en la Constitución, declarados como tal.

ii. La prueba ilícita puede resultar de la violación de derechos fundamentales -entendiendo derechos fundamentales como aquellos derechos subjetivos, libertades, inmunidades y potestades.

iii. La prueba ilícita es la que resulta de la violación de derechos fundamentales, pero no solamente, sino que también puede resultar de la vulneración de otras reglas jurídicas.

Precisamente, de este último punto tratará el siguiente epígrafe: la prueba ilícita como resultado de la violación de otras normas del ordenamiento jurídico.

\section{LA PRUEBA ILÍCITA COMO PRODUCTO DE LA VIOLACIÓN DE NORMAS JURÍDICAS}

La posición que define la prueba ilícita como aquella que resulta de la vulneración de normas del ordenamiento jurídico, y su impacto en el proceso, es el otro enfoque de los planteados en este análisis conceptual sobre la prueba ilícita. Para el estudio de esta posición se tomará en cuenta los criterios de varios autore ${ }^{28}$ que asumen postura en este sentido. Al mismo tiempo, se dará cuenta de los debates que se sostienen en la doctrina (en relación con los siguientes temas: tipos de normas vulneradas y principios), hasta llegar al examen de jurisprudencia que se exprese en esta línea y que sirva como referencia.

28 Martínez (2003, p. 81); Altavilla (1959, pp. 1-9); Silva (1963, pp. 68-70); Bonnier (1914, p. 64); Fenech (1960, p. 596); Cappelletti (1969, pp. 111-113); Serra (1991, p. 95); Véscovi (1997, p. 58); de Marino Borrego (1983, p. 613); Jorge Barreiro (1993, p. 1228). 
La idea principal que se sostiene acá es que el origen de la ilicitud de la prueba reside en que la misma ha sido obtenida mediante la violación de normas jurídicas, con independencia de la categoría de estas (constitucionales, legales, procesales o sustantivas, etc.) (Calderón, 2016, p. 140). Así lo afirman Gimeno Sendra, Moreno Catena y Cortés Domínguez, para quienes la prueba ilícita es aquella prueba que resulta de la infracción de cualquier ley (no solo la Constitución, sino también la legislación ordinaria) (1996, pp. 384-386).

Otro de los principales exponentes de esta postura es Véscovi (1970), quien plantea lo siguiente: “(...) la prueba ilícita puede provenir de la violación de una regla expresa, sea cual fuere su rango, legal, constitucional o reglamentaria, y también de la violación de normas no expresas" (p. 345).

Esta posición parte del argumento que explica que la prueba ilícita puede ser obtenida mediante la violación de normas constitucionales, legales o reglamentarias. Por ello, debe ser considerada inadmisible, como principio general del Derecho, aunque no exista una norma procesal expresa que proclame su nulidad (Véscovi, 1970, p. 360).

Desde este punto de vista, la prueba ilícita es entendida como aquella prueba que resulta de la violación de cualquier norma jurídica. Se puede afirmar que es una postura más amplia en cuanto a los posibles casos de ilicitud que puede abarcar. Además, se refiere a que es inadmisible una prueba producida de esta forma, dicha inadmisibilidad funciona como un principio general del derecho incluso para el caso que no exista una norma que declare la nulidad de estas pruebas. Considero que, efectivamente, ello funcionaría como un principio del derecho, en la medida en que los principios también suelen ser concebidos como implícitos en el ordenamiento jurídico ${ }^{29}$.

Sin embargo, es una posición que tiene algunos puntos discutibles. Veamos cuáles son. Por un lado, el punto relativo a la nulidad. Se está dando por hecho que la prueba que resulte de la violación de normas jurídicas debe declararse prueba nula. En ese caso faltaría la precisión sobre el tipo de nulidad al cual se estaría refiriendo (pero si es una nulidad que afecta derechos constitucionales, entonces suele considerarse que es absoluta). La nulidad puede ser absoluta o relativa: por nulidad absoluta se entiende aquella por la que los actos procesales no producen los efectos jurídicos, todo ello por no cumplirse los requisitos propios del acto, y por nulidad relativa o anulabilidad se entiende la que responde a que los vicios o irregularidades en el acto pueden ser subsanables ${ }^{30}$.

\footnotetext{
29 Compárese con Guastini (1999, p. 155) y Atienza y Ruiz Manero (1991, p. 112).

30 Véase Ortells (1999, pp. 255-256), y en otro de sus trabajos junto a Robles Garzón (2006, p. 29).
} 
Otro punto discutible es el de establecer la nulidad como única consecuencia para la prueba ilícita, cuando puede haber otras alternativas de solución como son las respuestas desde el derecho civil, del derecho de daños o hasta del derecho penal. Estas alternativas no conllevarían necesariamente a la nulidad del acto. Sin embargo, esta es una discusión sobre los efectos y, como ya se ha señalado, este será un punto por tratar en otra parte de la tesis. Basta con mostrar aquí que la conceptualización como ilícita de una prueba es una cuestión independiente de los efectos que deba tener esa ilicitud.

Frente a la posición general que concibe prueba ilícita como aquella que resulta de la violación de normas jurídicas, algunos autores introducen precisiones en cuanto al tipo de norma vulnerada (distinguiendo norma procesal de norma material). Esta postura sigue una lógica de Goldschmidt, pues establece la independencia y autonomía de las normas procesales respecto de las normas materiales o sustantivas.

La distinción entre norma procesal y norma material (o norma procesal y norma sustantiva) se utiliza mucho en la doctrina italiana. Dicha doctrina, partiendo de esa propia distinción, considera que son figuras diferentes la prueba ilícita y la prueba ilegítima: esta última, es considerada como aquella prueba obtenida contraviniendo normas jurídicas de carácter procesal, mientras que la prueba ilícita es aquella que resulta de la violación de normas jurídicas de tipo material o sustantivo ${ }^{31}$. Aunque es válido aclarar que esta distinción tan tajante se trata de un problema aún no resuelto ${ }^{32}$.

A mi manera de ver, la prueba ilícita es aquella prueba que resulta de la violación de normas jurídicas. Y es que, si nuestro análisis conceptual pretende ser lo suficientemente abarcador para los casos de prueba ilícita, bajo esta concepción se permiten incluir casos de ilicitud resultantes de la violación de derechos fundamentales y, además, los que resulten de la violación de otras normas jurídicas. Esta posición defendida tiene una mayor cobertura para dar cuenta de los casos paradigmáticos de prueba ilícita, pudiendo llegar incluso, hasta los casos de prueba ilícita resultantes de la violación de normas proce-

31 Galantini (1992, p. 83); Cordero (1961, pp. 32-34); Sainz de Robles y Albacar (1983, pp. 598 y ss.).

32 No queda clara la diferencia entre la norma sustantiva y la procesal. Al respecto, Passanante (2017), presenta la polémica de forma general, y se refiere al carácter esencialmente relativo de esta distinción, relatividad que aún y aceptada por la doctrina italiana, insisten en mantener la diferencia (pp. 87-103). Otros autores como Denti y Luiso (2015) se refieren a lo delicado del problema de establecer esta diferencia entre norma sustantiva y norma procesal, y que no está resuelto aún por la doctrina italiana (p. 4). 
dimentales (o administrativas, etc.) relativas a la cadena de custodia, donde se puede ver afectada la fiabilidad de la prueba.

Esta posición que entiende a la prueba ilícita como resultado de la vulneración de cualquier tipo de normas jurídicas tiene respaldo jurisprudencial. Desde la STC N 114/1984 de 29 de noviembre ${ }^{33}$ se valora que la ilicitud puede tener su origen no solo en la violación de derechos fundamentales, sino también en la infracción de la legalidad ordinaria, pero restringe la inadmisibilidad e ineficacia de la prueba a los supuestos en que la prueba haya sido obtenida con vulneración de derechos fundamentales.

El Tribunal Supremo de España ha dictado varias sentencias ${ }^{34}$ en consonancia con esta posición, distinguiendo cuando la ilicitud tiene por causa una violación de derechos fundamentales, o cuando es debida a la violación de normas de carácter ordinario:

(...) [C] uando el origen de la ilicitud de la prueba se encuentra en la violación de un derecho fundamental, no hay ninguna duda de que tal prueba carece de validez en el proceso y los Tribunales habrán de reputarla inexistente a la hora de construir la base fáctica en que haya de apoyarse una sentencia condenatoria. Otra cosa, quizá, haya que decir cuando la ilicitud sea de rango inferior, debiendo hacerse en cada caso una adecuada valoración de la norma violada en consideración a su auténtico y real fundamento y a su verdadera esencia y naturaleza (...) (Sentencia $N^{\circ} 2647$ del 29 de marzo de 1990 del Tribunal Supremo de España).

En este sentido, es válido subrayar que esta posición sobre prueba ilícita tiene apoyo jurisprudencial importante. Lo que sucede es que, una vez que se reconoce que es prueba ilícita, también aquella que deviene de ilicitudes de rango inferior al constitucional, entonces correspondería aclarar qué tipo

33 Sentencia N 114/1984, de 29 de noviembre del Tribunal Constitucional de España: “(...) la recepción de una prueba antijurídicamente lograda, no implica necesariamente lesión de un derecho fundamental, aunque se reconoce la posición preferente de los derechos fundamentales en el ordenamiento y de su afirmada condición de inviolables, con la imposibilidad de admitir en el proceso una prueba obtenida violentando éstos, pero también asegura que no se trata de decidir en general la problemática procesal de la prueba con causa ilícita, sino, más limitadamente, de constatar la "resistencia" frente a la misma de los derechos fundamentales (...)".

34 Sentencia $N^{\circ} 9350$ del 16 de diciembre de 1991 del Tribunal Supremo de España, dispone: “(...) por prueba ilegítimamente obtenida no debe entenderse tan solo aquella que se acomode a las exigencias constitucionales de no atentar, directa o indirectamente, contra los derechos fundamentales, sino también aquella que cumple las concretas garantías que para su práctica establece la legalidad procesal ordinaria (...)". Y en otras como la Sentencia N ${ }^{\circ}$ 817/2012 de 23 de octubre, la Sentencia N³170/2016 de 5 de julio, y la Sentencia N²286/2018 de 14 de junio. 
de consecuencias jurídicas cabría aplicar ante estos casos. Y este último punto sigue siendo un asunto pendiente en el orden no solo doctrinal, sino también jurisprudencial.

Hasta el momento se pudo analizar el problema relativo a los sentidos en que la doctrina y la jurisprudencia definen prueba ilícita. La primera posición se refiere a que es prueba ilícita aquella que resulta de la vulneración de derechos fundamentales, y la segunda postura entiende que la prueba ilícita no es sólo la que resulta de la violación de derechos fundamentales, sino que llega más allá, es prueba ilícita la obtenida o practicada mediante la violación de normas del ordenamiento jurídico en general. Siendo la segunda perspectiva la más adecuada para definir el fenómeno de la prueba ilícita.

Nos parece más adecuado este segundo sentido en que se define prueba ilícita, puesto que da la posibilidad de incluir mayor cantidad de casos relativos a esta. El único punto sería el de precisar las consecuencias o efectos que acarrearían.

Esta posición nos lleva a plantear un esquema en el cual cuando se vaya a definir prueba ilícita se ubiquen los siguientes tipos de casos:

i. Pruebas obtenidas y/o practicadas con vulneración de derechos fundamentales.

ii. Pruebas que resulten de la violación de otras normas del ordenamiento jurídico como las procesales, por ejemplo.

En cuanto a los tipos de casos previstos en el primer punto, las consecuencias que corresponden son las de nulidad del acto probatorio; mientras que para los tipos de casos previstos en el segundo punto, las consecuencias serían las de anulabilidad de los actos, o también llamados como nulidad relativa. Esto pues la nulidad absoluta va en respuesta a pruebas obtenidas y/o practicadas como resultados de actuar contrario a derechos fundamentales. El fundamento de esto último se encuentra en el argumento democrático del sistema que aludimos con anterioridad.

\section{CONCLUSIONES}

Una vez que se ha realizado este estudio sobre el concepto de prueba ilícita y sus principales problemas, se está en condiciones de poder concluir lo siguiente:

Primero. La prueba ilícita tiene su origen en los debates y decisiones de casos referidos a pruebas obtenidas mediante infracción de derechos funda- 
mentales. Todo lo cual tuvo lugar los contextos anglosajón y alemán de entre los siglos XVIII y XX, hasta nuestros días.

Segundo. Los sentidos en que se define la prueba ilícita son dos básicamente: i) es aquella prueba que se obtiene mediante violación de derechos y ii) es prueba que resulta de la infracción de normas del ordenamiento jurídico, entre las cuales también se incluyen los derechos fundamentales.

Tercero. Los argumentos que sustentan ambas definiciones tienen su base en concepciones democráticas unidas a la defensa de un Estado de Derecho y, además, en criterios relativos a la importancia que tiene una prueba ilícita en cuanto a la información que aporta al proceso.

Cuarto. Asumir una posición que defiende la segunda definición (ii) requiere continuar trabajando en el universo de casos que pueden englobarse ahí, pero al mismo tiempo se necesitaría trabajar en un estudio que profundice en las consecuencias jurídicas que correspondan según el tipo de casos y de ilicitud que se presente.

Quinto. Estudiar temas de esta naturaleza se vuelve un reto en el orden académico y profesional, dando pie a otras investigaciones dirigidas a explorar la regla de exclusión de la prueba ilícita y las alternativas a esta. Aunque dando por asumido el problema conceptual y sus principales análisis, tenemos una parte del camino recorrido.

\section{LISTA DE REFERENCIAS}

Alday, F. (2020). La exclusión de la Prueba llícita en España y México. Atelier. Ansuátegui, F. (2000). Las definiciones del estado de derecho y los derechos fundamentales. Sistema: revista de ciencias sociales, (158), 91-114.

Armenta Deu, T. (2009). La prueba ilícita (Un estudio comparado). Marcial Pons. Arozamena, C. (1999). Consideraciones generales sobre la prueba ilícita. Revista Actualidad Penal, (4), 83.

Asencio, J. M (1986). La prueba. Garantías constitucionales derivadas del artículo 24.2. Revista Poder Judicial, (4), 37-38.

Asencio, J. M. (1990). El proceso penal con todas las garantías. Revista lus et veritas, (33), 235-247.

http://revistas. pucp.edu.pe/index.php/iusetveritas/article/viewFile/12354/12918. Atienza, M. y Ruiz, J. (2000). Ilícitos atípicos. Editorial Trotta.

Beling, E. (1903). Die beweisverbote als grenzen der Wahrheitserforschung im Strafprozess. Wissenschaftliche Buchgessellschaft. 
Bustamante, R. (2018). El Derecho a probar como elemento esencial de un proceso justo. Ediciones Jurídicas Olejnik.

Calderón, E. (2016). La polémica de las ilicitudes probatorias derivadas de las fases de la cadena de custodia y el rango de la norma quebrantada. Derecho Global, (3), 131-159. https://doi.org/10.32870/dgedj.v0i3.13

Cappelletti, M. (1969). Proceso e ideología. II Mulino.

Fidalgo, C. (2003). Las "pruebas ilegales": de la exclusionary rule estadounidense al artículo 11.1 LOPJ. Estudios Constitucionales.

Gascón Abellán, M. (2006). Freedom of proof? El cuestionable debilitamiento de la regla de exclusión de la prueba ilícita. En Ferrer Beltrán, J., et. al (eds.), Estudios sobre la prueba (pp. 47-85). Instituto de Investigaciones Jurídicas de la Universidad Nacional Autónoma de México.

Gimeno Sendra, V; Moreno, V. y Cortés, V. (1996). Derecho procesal penal. Editorial Colex.

Gómez, J. L. (1985). El proceso penal alemán. Introducción y normas básicas. Bosch Editor.

Gómez, J. L. (1997). El proceso penal español. Tirant lo Blanch.

González, J. L. (1990) La prueba obtenida ilícitamente con violación de los derechos fundamentales (el derecho constitucional a la prueba y sus límites). Revista de Derecho Procesal, (1), 29-44.

Guariglia, F. (1996). “Las prohibiciones de valoración probatoria en el procedimiento penal". Jueces para la democracia, (25), 76-77.

Martínez, E. (2003). Eficacia de la prueba ilícita en el proceso penal. Editorial Tirant lo Blanch.

Miranda, M. (2013). El concepto de prueba ilícita y su tratamiento en el proceso penal. Especial referencia a la exclusionary rule estadounidense. UBIJUS.

Miranda, M. (2018). El concepto de prueba ilícita y su tratamiento en el proceso penal. Especial referencia a la exclusionary rule estadounidense. UBIJUS.

Nieva Fenoll, J. (2017). Policía judicial y prueba ilícita. Regla de exclusión y efecto disuasorio: un error de base. Diario La Ley, (9068), 1-35.

Parra, J. (1997). Pruebas ilícitas. Revista lus et Veritas, (14), 37-52. http://revistas. pucp.edu.pe/index.php/iusetveritas/article/view/15704/16140

Passanante, L. (2017). La prova illecita nel processo civile. G. Giappichelli Editore.

Peces-Barba, G. (1995). Curso de Derechos fundamentales. Teoría general.

Peláez, R. A. (2017). La prueba ilícita desde la perspectiva de la regla de exclusión y su aplicación en el proceso civil. Universidad Externado de Colombia. 
Picó i Junoy, J. (1996). El derecho a la prueba en el proceso civil. Bosch Editor. Picó i Junoy, J. (1997). Las garantías constitucionales del proceso. Bosch Editor. Sainz de Robles, F. y Albacar, L. (1983). El derecho a la prueba. En Primeras Jornadas de Derecho Judicial. Presidencia del Tribunal Supremo, Secretaría Técnica.

Silva, V. (1963). La prueba procesal. Tomo I. Editorial Revista de Derecho Privado. Véscovi, E. (1970). Premisas para la consideración del tema de la prueba ilícita. Revista de Derecho Procesal Iberoamericana, (2), 345. 